\title{
Neuroprotective effects of Rhizoma Dioscoreae polysaccharides against neuronal apoptosis induced by in vitro hypoxia
}

\author{
QIN XIANG ${ }^{1}$, WEN-YUN ZHOU ${ }^{2}$, WEI-XU HU ${ }^{3}$, ZHU WEN $^{4}$, DAN HE $^{2}$, XIAO-MU WU ${ }^{2}$, \\ HUI-PING WEI ${ }^{2}$, WEN-DING WANG ${ }^{2}$ and GUO-ZHU HU ${ }^{2}$
}

\begin{abstract}
${ }^{1}$ State Key Laboratory of Chemo/Biosensing and Chemometrics, College of Biology, Hunan University, Changsha, Hunan 410082; ${ }^{2}$ Institute of Clinical Medical Sciences, Jiangxi Province People's Hospital, Nanchang, Jiangxi 330006; ${ }^{3}$ Department of Radiotherapy, Zhongshan Hospital, Fudan University, Shanghai 200032; ${ }^{4}$ Department of Hematology, Jiangxi Academy of Medical Science, Nanchang, Jiangxi 330006, P.R. China
\end{abstract}

Received January 16, 2015; Accepted September 25, 2015

DOI: 10.3892/etm.2015.2819

\begin{abstract}
Rhizoma Dioscoreae polysaccharides (RDPS) are the primary active ingredient of Rhizoma Dioscoreae, which is a traditional Chinese medicine. RDPS have previously been shown to scavenge reactive oxygen species, and protect against D-galactose-induced mimetic aging. The present study aimed to investigate the neuroprotective effects of RDPS against hypoxia-induced neuronal cell apoptosis. Neuronal cells harvested from pregnant Sprague-Dawley rats were divided into groups, as follows: i) Normal control group; ii) hypoxia-induced apoptosis neuronal cell model; iii) $0.025 \mathrm{~g} / \mathrm{l}$ RDPS-treated group; iv) $0.05 \mathrm{~g} / \mathrm{l}$ RDPS-treated group; v) $0.1 \mathrm{~g} / 1 \mathrm{RDPS}$-treated group; and vi) $0.25 \mathrm{~g} / \mathrm{l}$ RDPS treated group. Neuronal cell viability was investigated using an MTT assay, and neuronal cell apoptosis was analyzed using Annexin V-fluorescein isothiocyanate/propidium iodide double-staining, Hoechst 33342 fluorescent staining, Rhodamine 123 staining, polymerase chain reaction and immunocytochemical staining. The RDPS-treated neuronal cells exhibited improved viability, and decreased hypoxia-induced mitochondrial injury and apoptosis. In addition, the mRNA and protein expression levels of caspase-3 and B-cell lymphoma (Bcl)-2-associated $\mathrm{X}$ protein (Bax) were significantly downregulated, whereas the mRNA and protein expression levels of Bcl-2 were significantly upregulated, in the RDPS-treated hypoxic neurons, as compared with the apoptosis model $(\mathrm{P}<0.05)$. Furthermore, the ratio of $\mathrm{Bcl}-2$ expression:Bax expression significantly increased following RDPS treatment, as compared with the apoptosis model $(\mathrm{P}<0.05)$. The results of the present study suggested that RDPS may attenuate hypoxia-induced neuronal cell apoptosis by altering the expression levels of key apoptosis-regulating proteins in hypoxic neurons.
\end{abstract}

Correspondence to: Professor Guo-Zhu $\mathrm{Hu}$, Institute of Clinical Medical Sciences, Jiangxi Province People's Hospital, 152 Ai Guo Road, Nanchang, Jiangxi 330006, P.R. China

E-mail: hgz56@126.com

Key words: apoptosis, Rhizoma Dioscoreae, hypoxia, in vitro, neurons, polysaccharides

\section{Introduction}

Rhizoma Dioscoreae (RD) is a traditional Chinese medicine described in the Pharmacopoeia of the People's Republic of China, of which the RD polysaccharides (RDPS) are the major active ingredient (1). Ko and Hong (2) demonstrated the safety of using Dioscoreae rhizome in the practice of pharmacopuncture, and various studies have detected therapeutic effects for RDPS. In particular, RDPS administered intragastrically was shown to decrease the levels of malondialdehyde (MDA), nitric oxide synthase and nitric oxide, alleviate liver inflammation, and decrease the liver index and alanine transaminase activity in a mouse model of chemokine (C-C motif) ligand-4-induced liver injury (3). Furthermore, treatment of a mouse model of D-galactose-induced mimetic aging with RDPS was associated with increased superoxide dismutase (SOD), glutathione peroxidase (GSH-Px) and $\mathrm{Na}^{+} / \mathrm{K}^{+}$-ATPase activities, as well as decreased serum levels of MDA $(4,5)$. In rats, focal application of Dioscoreae rhizome extract at a sciatic nerve crush injury site was associated with increased levels of the axonal growth-associated protein and cyclin-dependent kinase-1 in the distal portion of the injured nerve (6). Furthermore, previous in vitro studies have suggested that RDPS is able to scavenge 1,1-diphenyl-2-picrylhydrazyl, $\mathrm{OH}$ and $\mathrm{O}_{2}^{-}$free radicals $(7,8)$.

In acute or chronic ischemia/hypoxia, the necrosis and apoptosis of neurons is mediated by the production of reactive oxygen species (ROS) or activation of the mitochondrial apoptosis pathway $(9,10)$. The present study aimed to investigate the neuroprotective effects of RDPS against in vitro hypoxia-induced cerebral cortical neuron apoptosis. The results of the present study suggested that RDPS was able to improve neuronal cell viability, and inhibit hypoxia-induced apoptosis of neuronal cells.

\section{Materials and methods}

Materials. Gibco neurobasal medium and B-27 supplement were obtained from Thermo Fisher Scientific, Inc. (Waltham, MA, USA). Equine serum, poly-D-lysine and trypsin were obtained from Sigma-Aldrich (St. Louis, MO, USA). 
RDPS (extracted using a mixture of distilled water, chloroform, n-butanol and ethanol, and diluted with neurobasal medium; polysaccharide content, $>95.0 \%$ ) was purchased from Nanjing Zelang Medical Technological Co., Ltd. (Nanjing, China). Hoechst 33342, Annexin V-fluorescein isothiocyanate (FITC) and Rhodamine 123 staining kits were obtained from Nanjing KeyGen Biotech Co., Ltd. (Nanjing, China). MTT was obtained from Beijing Probe Biotech Co., Ltd. (Beijing, China). TransScript ${ }^{\mathrm{TM}}$ two-step reverse transcription-polymerase chain reaction (RT-PCR) Supermix kit was obtained from Beijing TransGen Biotech Co., Ltd. (Beijing, China).

Rats. A total of 52 pregnant Sprague-Dawley rats were bred and housed at the Laboratory Animal Services Centre of the Jiangxi College of Traditional Chinese Medicine (Nanchang, China). The rats were house in a room that was free of noise and strong odors, with a controlled temperature of $23 \pm 2{ }^{\circ} \mathrm{C}$ and $60 \pm 5 \%$ relative humidity, and were maintained in a $12 \mathrm{~h}$ light/12 $\mathrm{h}$ dark cycle. The rats had free access to water and food. All experiments were performed in accordance with the animal experimental guidelines established by the Ministry of Science and Technology of the People's Republic of China, and were approved by the ethics committee of Jiangxi Province People's Hospital (Nanchang, China).

\section{Cytotoxicity of RDPS}

Cerebral cortical neurons in primary serum-free culture. A neuronal suspension was prepared from the pregnant rats, as outlined previously (11). Briefly, the pregnant rats were anesthetized with $1.5 \mathrm{ml} 10 \%$ chloral hydrate (Sangon Biotech Co., Ltd., Shanghai, China) and then fixed on the animal operating table. Their abdominal skin was disinfected with a $75 \%$ alcohol gauze and laparotomy was performed. The fetal rats were carefully removed from the uterus and their brain was removed following removal of the scalp and skull. The brain tissues were placed in cold D-Hank's solution (Gibco; Thermo Fisher Scientific, Inc.) containing $4.5 \%$ glucose in a petri dish. Subsequently, the fetal rats meninges and blood vessels were removed under a Leica S6 E stereomicroscope (Leica Microsystems, Wetzlar, Germany), and the brain cortex tissue was isolated and cut into $1 \times 1 \times 1 \mathrm{~mm}$ tissue sections. The sections were then placed into a $0.25 \%$ pancreatic enzyme EDTA solution at $37^{\circ} \mathrm{C}$ for $20 \mathrm{~min}$, followed by termination of digestion by addition of $5 \mathrm{ml}$ Dulbecco's Modified Eagle medium (DMEM; Gibco, Thermo Fisher Scientific, Inc.) supplemented with $10 \%$ horse serum and $10 \%$ fetal bovine serum for $5 \mathrm{~min}$. The cells were isolated from the sections by a mechanical method using a Pasteur pipette, and passed through a 200 mesh stainless steel sieve. Next, the cells were counted and adjusted to a concentration of $5 \times 10^{6} / \mathrm{ml}$ using DMEM.

Subsequently, $0.1 \mathrm{ml}$ cells were seeded at a density of $5 \times 10^{5}$ cells $/ \mathrm{ml}$ into 96 -well culture plates coated with polylysine, and were subsequently stored in a $5 \% \mathrm{CO}_{2}$ incubator (Thermo Fisher Scientific, Inc.) at $37^{\circ} \mathrm{C}$ with saturated humidity. After 4 h, Gibco Dulbecco's modified Eagle's medium (Thermo Fisher Scientific, Inc.), supplemented with $10 \%$ equine serum, was removed from the plates, and the cell cultures were incubated with $2.0 \%$ B-27 neurobasal medium for 4 days. Subsequently, the cells were incubated with RDPS $(0.025,0.05,0.10,0.25$, $0.50,1.0,2.0,4.0$ or $8.0 \mathrm{~g} / \mathrm{l})$ for $48 \mathrm{~h}$, after which the culture medium $(0.1 \mathrm{ml})$ was removed and added to 96 -well plates containing $0.5 \% \mathrm{MTT}$ for $4 \mathrm{~h}$. Following removal of the culture media, $0.15 \mathrm{ml}$ dimethyl sulfoxide (DMSO; Sigma-Aldrich) was added to the wells, and the plates were agitated for $10 \mathrm{~min}$. Absorbance [optical density (OD)] values were measured at $490 \mathrm{~nm}$ using a microplate reader (ELx800 ${ }^{\mathrm{TM}}$; BioTek Instruments, Inc., Winooski, VT, USA).

Cerebral cortical neurons in primary serum-free hypoxia/reoxygenation culture. Cytotoxicity and MTT analyses were conducted as outlined previously (11). Briefly, the neurons were treated with RDPS $(0.025,0.05,0.10,0.25$, $0.50,1.0$ or $2.0 \mathrm{~g} / \mathrm{l}$ ) for $4 \mathrm{~h}$, after which the 96 -well plates were stored for $12 \mathrm{~h}$ in a hypoxia incubator (YQX-II Anaerobic Incubator; Shanghai Hengyue Medical Instruments Co., Ltd, Shanghai, China), containing $85 \%$ nitrogen, $10 \%$ hydrogen and $5 \% \mathrm{CO}_{2}$, at $37^{\circ} \mathrm{C}$. Subsequently, the plates were transferred to a $5 \% \mathrm{CO}_{2}$ reoxygenation incubator for $24 \mathrm{~h}$, after which the culture media $(0.1 \mathrm{ml})$ was removed and added to 96 -well plates containing $0.5 \%$ MTT for $4 \mathrm{~h}$. Subsequently, the culture media was removed, $0.15 \mathrm{ml}$ DMSO was added to the wells, the plates were agitated for $10 \mathrm{~min}$, and the $\mathrm{OD}$ values were measured at $490 \mathrm{~nm}$ using a microplate reader.

Grouping. The harvested rat neuronal cells were divided into five groups, as follows: i) Normal control group (C), in which the neurons $\left(5 \times 10^{5}\right.$ cells $\left./ \mathrm{ml}\right)$ were cultured in an incubator at $37^{\circ} \mathrm{C}$, containing $5 \% \mathrm{CO}_{2}$ and saturated humidity for 6 days; ii) apoptosis model group (A), in which neurons $\left(5 \times 10^{5}\right.$ cells $\left./ \mathrm{ml}\right)$ were cultured in an incubator at $37^{\circ} \mathrm{C}$, containing $5 \% \mathrm{CO}_{2}$ and saturated humidity for 4 days, after which the neurons were placed in a hypoxia incubator for $12 \mathrm{~h}$, followed by transfer to a $5 \% \mathrm{CO}_{2}$ reoxygenation incubator for $24 \mathrm{~h}$; iii) $0.025 \mathrm{~g} / 1$ RDPS-treated group (RDPS1); iv) $0.05 \mathrm{~g} / 1$ RDPS-treated group (RDPS2); v) 0.1 g/l RDPS-treated group (RDPS3); and vi) $0.25 \mathrm{~g} / 1$ RDPS-treated group (RDPS4). Generation of the RDPS-treated groups involved culturing the rat neurons $\left(5 \times 10^{5}\right.$ cells $\left./ \mathrm{ml}\right)$ in an incubator containing $5 \% \mathrm{CO}_{2}$ at $37^{\circ} \mathrm{C}$, with saturated humidity for 4 days, after which the cells were incubated with the appropriate concentration of RDPS for $4 \mathrm{~h}$. Subsequently, the neurons were cultured under hypoxic conditions for $12 \mathrm{~h}$, and then incubated for $24 \mathrm{~h}$ in a $5 \% \mathrm{CO}_{2}$ reoxygenation incubator.

Hoechst 33342 fluorescence staining. Hoechst 33342 fluorescence staining was conducted as outlined previously (11). The apoptotic neurons were observed using fluorescence microscopy. Briefly, the neurons ( $>200)$ were counted randomly under a high power microscope (DMI 3000; Leica Microsystems $\mathrm{GmbH}$, Wetzlar, Germany) and the apoptotic rate was calculated as follows: Apoptotic rate $(\%)=$ (number of apoptotic neurons/total number of neurons) x $100 \%$.

Annexin V FITC/propidium iodide (PI) double staining and flow cytometric analysis. The neurons were digested using $0.02 \%$ ethylenediaminetetraacetic acid (EDTA) and $0.125 \%$ pancreatin solution (Sigma-Aldrich), and the resulting neuronal cell suspension was centrifuged for $5 \mathrm{~min}$ at $300 \mathrm{x} \mathrm{g}$, after which the supernatant was removed. The neurons were washed twice with phosphate-buffered saline (PBS), followed by centrifugation for an additional $5 \mathrm{~min}(300 \mathrm{x}$ g). The cells 
(1-5x $10^{5}$ cells $\left./ \mathrm{ml}\right)$ were suspended in $500 \mu 1$ binding buffer, after which $5 \mu \mathrm{l}$ Annexin-FITC and $5 \mu \mathrm{l}$ PI was added, with agitation. The cells were incubated at room temperature in the dark for $10 \mathrm{~min}$, followed by centrifugation for $5 \mathrm{~min}$ at $300 \mathrm{x}$ g. Subsequently, the labeling liquid was removed and the cells were washed once with incubation buffer. The cells were analyzed in a flow cytometer (Coulter Epics xL; Beckman Coulter Inc., Brea, CA, USA) with argon ion laser-excited fluorescence at $488 \mathrm{~nm}$. Flowjo 7.6 software was used to analyze the results of the flow cytometric analysis (Tree Star Inc., Ashland, OR, USA).

Rhodamine 123 staining and flow cytometric analysis. Rhodamine 123 staining was performed according to the manufacturer's protocol. Briefly, hypoxia/reoxygenation cultured neurons were digested using $0.02 \%$ EDTA and $0.125 \%$ pancreatin solution, and the resulting neuronal cell suspension was centrifuged for $5 \mathrm{~min}$ at $300 \mathrm{x} \mathrm{g}$, after which the supernatant was removed. The neurons were washed with PBS three times and Rhodamine 123 dye (final concentration, $0.005 \mathrm{~g} / \mathrm{l}$ ) was added. Following incubation for $20 \mathrm{~min}$, the neurons were washed three times with PBS and incubated for $60 \mathrm{~min}$. Subsequently, the neurons were collected and analyzed in a flow cytometer with argon ion laser-excited fluorescence at $488 \mathrm{~nm}$. Flowjo 7.6 software was used to analyze the fluorescence intensity.

Semi-quantitative PCR assay. The PCR assay (MyCycler ${ }^{\mathrm{TM}}$ Thermal Cycler; Bio-Rad Laboratories, Inc., Hercules, CA, USA) was performed according to the manufacturer's protocol. Briefly, total mRNA extraction was performed as follows: TRIzol ${ }^{\circledR}$ reagent (Invitrogen; Thermo Fisher Scientific, Inc.) extraction for $5 \mathrm{~min}$, followed by chloroform treatment for $2 \mathrm{~min}$, centrifugation at $12,000 \mathrm{x}$ g for $15 \mathrm{~min}$, isopropyl alcohol treatment for $20 \mathrm{~min}$, followed by further centrifugation at $12,000 \mathrm{x} g$ for $10 \mathrm{~min}$. The supernatant was removed and $75 \%$ ethanol precipitation was performed, followed by centrifugation at 7,500 x g for $5 \mathrm{~min}$, supernatant removal and air drying. Diethylpyrocarbonate-treated water was then added, in order to dissolve the mRNA, at $65^{\circ} \mathrm{C}$ for $10-15 \mathrm{~min}$. The OD value of the RNA was measured at $260 \mathrm{~nm}$ using a SmartSpec Plus ultraviolet spectrophotometer (Bio-Rad Laboratories, Inc.).

The RNA OD value was used to calculate the concentration of RNA, as follows: RNA concentration $(\mathrm{mg} / \mathrm{ml})=40 \times \mathrm{OD}_{260}$ value $\mathrm{x}$ dilution ratio/1,000. Reverse transcription of mRNA into cDNA was performed as follows: Total mRNA $(3 \mu \mathrm{l})$, $1 \mu \mathrm{l}$ random primer $(0.1 \mu \mathrm{g} / \mathrm{ml}), 10 \mu \mathrm{l} 2 \mathrm{X}$ TS Reaction mix, $1 \mu \mathrm{l}$ TransScript $^{\mathrm{TM}}$ RT/RI Enzyme mix and $5 \mu \mathrm{l}$ ribonuclease-free water, was mixed and incubated at $25^{\circ} \mathrm{C}$ for $10 \mathrm{~min}, 42^{\circ} \mathrm{C}$ for $30 \mathrm{~min}$ and $85^{\circ} \mathrm{C}$ for $5 \mathrm{~min}$.

The $\beta$-actin, caspase-3, B-cell lymphoma 2 (Bcl-2) and $\mathrm{Bcl}-2$-associated $\mathrm{X}$ protein (Bax) genes were amplified according to the following protocol: The cDNA $(3 \mu 1)$, $1 \mu \mathrm{l}$ forward primer $(10 \mu \mathrm{M}), 1 \mu \mathrm{l}$ reverse primer $(10 \mu \mathrm{M})$, $25 \mu 12 \mathrm{X}$ TransTap ${ }^{\mathrm{TM}}$ HiFi PCR SuperMix II and $20 \mu$ l double distilled $\mathrm{H}_{2} \mathrm{O}$, were mixed and subjected to $32 \mathrm{PCR}$ cycles $\left(94^{\circ} \mathrm{C}\right.$ for $5 \mathrm{~min}, 94^{\circ} \mathrm{C}$ for $30 \mathrm{sec}, 55^{\circ} \mathrm{C}$ for $30 \mathrm{sec}, 72^{\circ} \mathrm{C}$ for $1 \mathrm{~min}$ and $72^{\circ} \mathrm{C}$ for $10 \mathrm{~min}$ ) for $\beta$-actin and caspase-3, and $32 \mathrm{PCR}$ cycles $\left(94^{\circ} \mathrm{C}\right.$ for $5 \mathrm{~min}, 94^{\circ} \mathrm{C} 30 \mathrm{sec}$, and $58^{\circ} \mathrm{C}$ for $30 \mathrm{sec}, 72^{\circ} \mathrm{C} 1 \mathrm{~min}$ and $72^{\circ} \mathrm{C}$ for $10 \mathrm{~min}$ ) for Bax and $\mathrm{Bcl}-2$.
Agarose gel electrophoresis was performed using $5 \mu \mathrm{l}$ of the PCR products at $120 \mathrm{~V}$ for $45 \mathrm{~min}$. The gray-scale value of each DNA band was measured using Quantity One software (Bio-Rad Laboratories, Inc.). The levels of gene expression were quantified by calculating the ratio of the OD values of the respective gene:OD value of the internal control.

The gene primer sequences were as follows: $\beta$-actin (432bp) forward, 5'-TCAGGTCATCACTATCGGCAAT-3' and reverse, 5'-AAAGAAAGGGTGTAAAACGCA-3'; caspase-3 (159bp) forward, 5'-GCATGCCATATCATCGTCAG-3' and reverse, 5'-GGACCTGTGGACCTGAAAAA-3'; Bax (173 bp) forward, 5'-GATCAGCTCGGGCACTTTAG-3' and reverse, 5'-TGCAGAGGATGATTGCTGAC-3'; and Bcl-2 (223bp) forward, 5'-ATGCCGGTTCAGGTACTCAG-3' and reverse, 5'-CGACTTTGCAGAGATGTCCA-3'.

Immunocytochemical staining. Immunocytochemical staining was performed, as outlined previously (11). Briefly, the cells were incubated with rabbit anti-rat Bcl-2 (dilution, 1:200; cat. no. D2010), rabbit anti-rat Bax (dilution, 1:200; cat. no. 12910), or rabbit anti-caspase-3 (dilution, 1:400; cat. no. E1410) polyclonal primary antibodies, followed by incubation with a goat anti-rabbit immunoglobulin G secondary antibody (cat. no. 202012; all antibodies were purchased from Zhongshan Golden Bridge Biotechnology, Beijing, China). The cells that were brown in appearance under a light microscope (BX43; Olympus Corp., Tokyo, Japan) were designated positive, whereas unstained or 'buffy' cells were considered negative. A total of 200 cells were randomly counted in order to calculate the positive rate, as follows: Positive rate $(\%)=($ the number of positive cells/total number of cells) x $100 \%$.

Statistical analysis. Data are presented as the mean \pm standard deviation. Experimental data that conformed to a normal distribution and homogeneity of variance were analyzed using a one-way analysis of variance, and post hoc tests were used for comparison between two groups. Experimental data that did not fit a normal distribution or homogeneity of variance were analyzed using a non-parametric test. $\mathrm{P}<0.05$ was considered to indicate a statistically significant difference. For statistical analysis, the SPSS version 17.0 software (SPSS, Inc., Chicago, IL, USA) was used.

\section{Results}

Effects of RDPS on cultured cerebral cortical neuronal cell viability. The cultured neurons were treated with RDPS for 48 h. MTT detection demonstrated that the neuronal cell viability was markedly improved following treatment with 0.025-2.0 g/l RDPS, with significant improvements being detected for cells treated with $0.05-0.5 \mathrm{~g} / 1$, as compared with the normal control ( $\mathrm{P}<0.05$; Fig. 1). However, cytotoxicity was detected following treatment of the neuronal cells with $8.0 \mathrm{~g} / 1$ RDPS ( $\mathrm{P}<0.05$; Fig. 1).

Effects of RDPS on hypoxia/reoxygenation-cultured cerebral cortical neuronal cell viability. The cultured neurons were treated with RDPS for $48 \mathrm{~h}$, after which the cells were cultured under hypoxic conditions for $12 \mathrm{~h}$, followed by culturing under reoxygenating conditions for $24 \mathrm{~h}$. The neuronal cell survival rate 


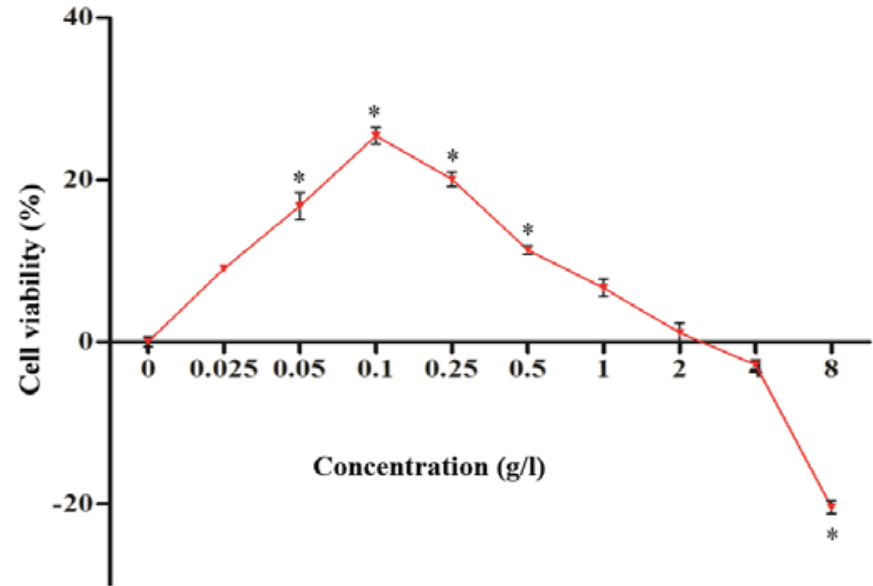

Figure 1. Effects of RDPS on the viability of cultured cerebral cortical neuronal cells, measured as OD values. Neuronal cells were treated with various concentrations of RDPS. The OD value for the normal control group was set to $0 \%$. Proliferation rates were calculated according to the ratio of the OD value of a specific RDPS concentration group and the OD value of the control group. Data are presented as the mean \pm standard deviation $(n=5$ separate cultures). $\mathrm{P}<0.05$ vs. the control group. RDPS, Rhizoma Dioscoreae polysaccharides; OD, optical density.

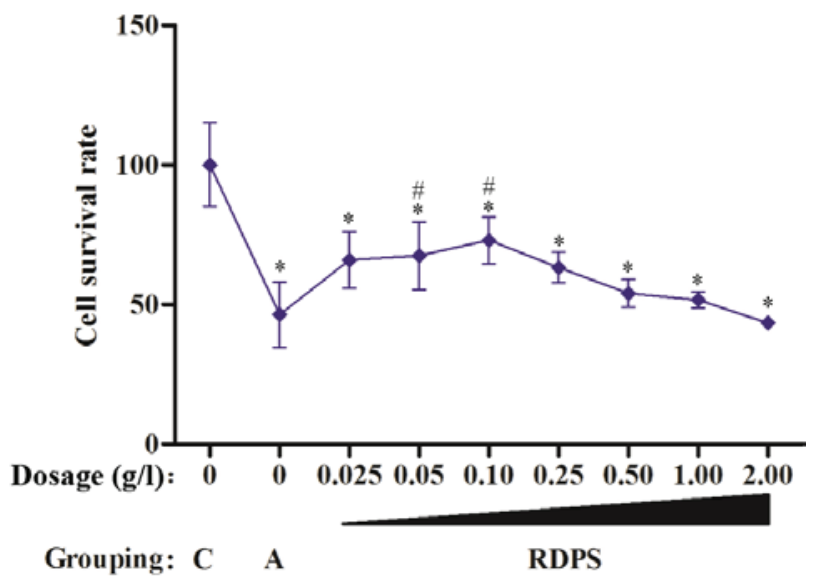

Figure 2. Effects of RDPS on the hypoxia/reoxygenation-cultured cerebral cortical neuronal cell survival rate. Neuronal cells were treated with various concentrations of RDPS. The OD value for the normal control group was set to $100 \%$ proliferation. Proliferation rates for the hypoxia/reoxygenation cerebral cortical neurons were calculated according to the ratio of the OD value of the apoptosis model or the various RDPS concentration groups and the OD value of the normal control group. Data are presented as the mean \pm standard deviation ( $\mathrm{n}=5$ separate cultures). ${ }^{*} \mathrm{P}<0.05$ vs. the control; ${ }^{\#} \mathrm{P}<0.05$ vs. the apoptosis model. RDPS, Rhizoma Dioscoreae polysaccharides; OD, optical density.

was markedly improved following treatment with $0.025-1.0 \mathrm{~g} / \mathrm{l}$ RDPS, and was significantly improved following treatment with 0.05-0.10 g/l RDPS, as compared with the apoptosis model ( $\mathrm{P}<0.05$; Fig. 2). These results suggest that $0.05-0.10 \mathrm{~g} / \mathrm{l}$ RDPS may protect neuronal cells against hypoxia-induced apoptosis.

Effects of RDPS on hypoxia/reoxygenation-induced cerebral cortical neuronal cell apoptosis. The neurons were treated with RDPS for $4 \mathrm{~h}$, after which the cells were cultured under hypoxic conditions for $12 \mathrm{~h}$ and under reoxygenating conditions for $24 \mathrm{~h}$. Hoechst 33342 fluorescence staining demonstrated that treatment of the neuronal cells with 0.025-0.25 g/1 RDPS, and particularly with $0.10 \mathrm{~g} / \mathrm{l}$ RDPS, significantly decreased

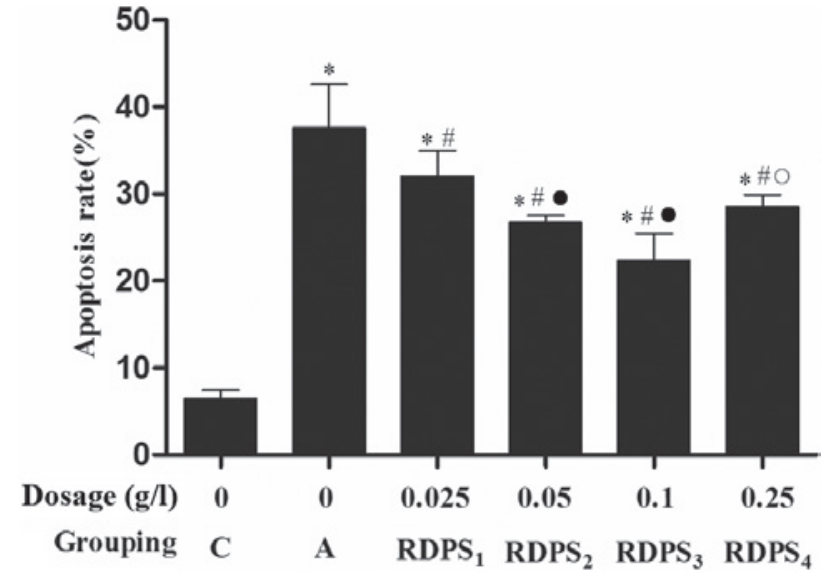

Figure 3. Effects of RDPS against hypoxia/reoxygenation-induced neuronal cell apoptosis were measured using Hoechst 33342 fluorescence staining. The neuronal cells were treated with various concentrations of RDPS and were seeded into 96-well plates, after which $>200$ cells/well were counted under a high power microscope. The apoptotic rate was calculated as the number of apoptotic neurons/the total number of neurons $\mathrm{x} 100 \%$. The data are presented as the mean \pm standard deviation ( $\mathrm{n}=3$ separate cultures). ${ }^{*} \mathrm{P}<0.05$ vs. normal control group; ${ }^{\#} \mathrm{P}<0.05$ vs. the apoptosis model group; ${ }^{\mathrm{P}}<0.05$ vs. RDPS1 group; ${ }^{\circ} \mathrm{P}<0.05$ vs. the RDPS3 group. $\mathrm{C}$, the normal control group; A, apoptosis model group; RDPS, Rhizoma Dioscoreae polysaccharides.

the apoptotic rate, as compared with the apoptosis model group ( $\mathrm{P}<0.05$; Fig. 3$)$.

Annexin V/PI double staining demonstrated that treatment with 0.025-0.25 g/l RDPS, in particular with $0.10 \mathrm{~g} / 1$, significantly decreased the early apoptotic rate, as compared with the apoptosis model group $(\mathrm{P}<0.05$; Fig. 4A); however, there were no marked differences in the rates of apoptosis at the late apoptotic stage between the various RDPS-treated groups, as compared with that in the apoptosis model group ( $\mathrm{P}>0.05$; Fig. 4B).

Treatment of the hypoxic neurons with $0.025-0.25 \mathrm{~g} / 1$ RDPS, and particularly $0.10 \mathrm{~g} / 1$ RDPS, significantly increased the mean fluorescence intensity (MFI) of Rhodamine 123 staining, as compared with the apoptosis model group $(\mathrm{P}<0.05$; Fig. 5). These results suggest that treatment with RDPS attenuates hypoxia-induced mitochondrial injury in neuronal cells.

PCR demonstrated that the caspase-3 mRNA expression levels in hypoxic neurons treated with 0.10-0.25 g/l RDPS, and the Bax mRNA expression levels in hypoxic neurons treated with 0.05-0.25 g/l RDPS, were significantly decreased, as compared with the apoptosis model group $(\mathrm{P}<0.05$; Figs. 6 and 7). Conversely, the Bcl-2 mRNA expression levels in hypoxic neurons treated with 0.05-0.10 g/1 RDPS were significantly increased, as compared with the apoptosis model group $(\mathrm{P}<0.05$; Figs. 6 and 7). Following treatment with 0.05-0.10 g/l RDPS, the ratio of Bcl-2 mRNA:Bax mRNA was significantly increased in the neurons cultured under hypoxia/reoxygenation conditions, as compared with the apoptosis model group ( $\mathrm{P}<0.05$; Fig. 6). These results suggest that decreased hypoxia-induced neuronal cell apoptosis following treatment with RDPS may be due to a reduction in the expression levels of apoptosis-regulating genes.

Immunocytochemical staining indicated that the number of caspase-3-positive cells in the hypoxic neurons treated with 0.025-0.25 g/l RDPS (in particular those treated with $0.10 \mathrm{~g} / \mathrm{l}$ ), 

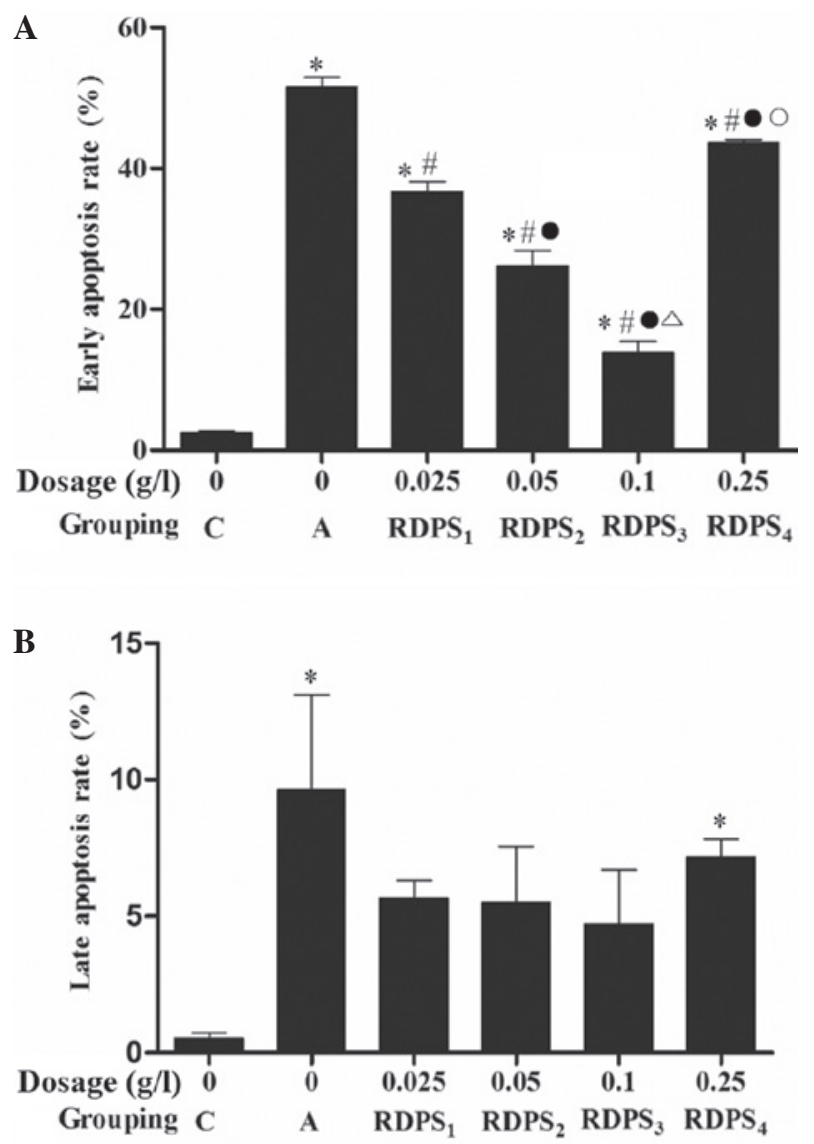

Figure 4. Effects of RDPS on hypoxia/reoxygenation-induced neuronal cell apoptotic rates in (A) early- and (B) late-stage apoptosis were analyzed using Annexin V/PI double staining and flow cytometric detection. The neuronal cells were treated with $0,0.025,0.05,0.10$ or $0.25 \mathrm{~g} / 1 \mathrm{RDPS}$. Data are presented as the mean \pm standard deviation ( $\mathrm{n}=3$ cultures). ${ }^{*} \mathrm{P}<0.05$ vs. the normal control group; ${ }^{*} \mathrm{P}<0.05$ vs. the apoptosis model group; ${ }^{\mathrm{P}} \mathrm{P}<0.05$ vs. the RDPS1 group; ${ }^{\circ} \mathrm{P}<0.05$ vs. the RDPS 3 group; ${ }^{\triangle} \mathrm{P}<0.05$ vs. the RDPS2 group. $\mathrm{C}$, the normal control group; A, the apoptosis model group; RDPS, Rhizoma Dioscoreae polysaccharides;PI, propidium iodide.

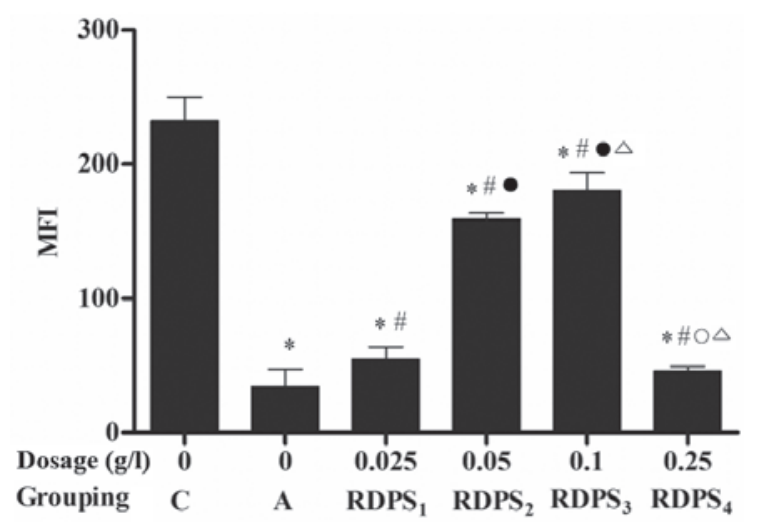

Figure 5. Effects of RDPS against hypoxia-induced mitochondrial injury in neuronal cells. Neuronal cells cultured under hypoxic conditions for $12 \mathrm{~h}$ and under reoxygenating conditions for $24 \mathrm{~h}$, were treated with $0,0.025,0.05,0.10$ or $0.25 \mathrm{~g} / 1$ RDPS. The ability of RDPS to protect against hypoxia-induced mitochondrial injury was investigated using Rhodamine 123 staining and flow cytometry. Data are presented as the mean \pm standard deviation of triplicate experiments. ${ }^{\text {}} \mathrm{P}<0.05$ vs. the normal control group; ${ }^{*} \mathrm{P}<0.05$ vs. the apoptosis model group; ${ }^{\mathrm{P}} \mathrm{P}<0.05$ vs. the RDPS 1 group; ${ }^{\circ} \mathrm{P}<0.05$ vs. the RDPS 3 group; ${ }^{\wedge} \mathrm{P}<0.05$ vs. the RDPS2 group. MFI, mean fluorescence intensity; $\mathrm{C}$, the normal control group; A, the apoptosis model group; RDPS, Rhizoma Dioscoreae polysaccharides.
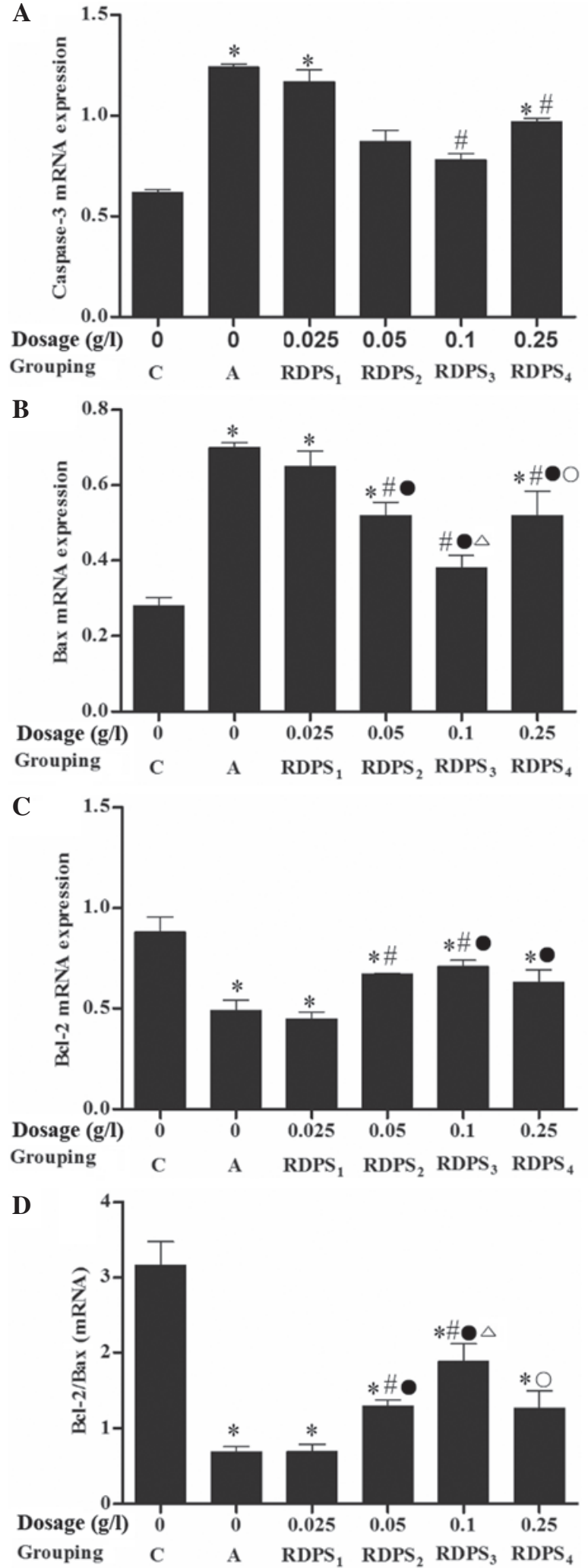

Figure 6. Effects of RDPS on mRNA expression levels of (A) caspase-3, (B) Bax (C) Bcl-2 and (D) Bcl-2/Bax in the hypoxic neurons. Neuronal cells cultured under hypoxic conditions for $12 \mathrm{~h}$ and under reoxygenating conditions for $24 \mathrm{~h}$, were treated with RDPS. Expression levels of key regulators of apoptosis were analyzed using polymerase chain reaction and agarose gel electrophoresis. The gene expression levels were semi-quantified by calculating the ratio of the OD values of the respective genes vs. the OD value of the internal control ( $\beta$-actin). Data are presented as the mean \pm standard deviation of triplicate experiments. ${ }^{*} \mathrm{P}<0.05$ vs. normal control group; ${ }^{*} \mathrm{P}<0.05$ vs. apoptosis model group; ${ }^{\circ} \mathrm{P}<0.05$ vs. RDPS1 group; ${ }^{\circ} \mathrm{P}<0.05$ vs. RDPS3; ${ }^{\Delta} \mathrm{P}<0.05$ vs. RDPS2 group. RDPS, Rhizoma Dioscoreae polysaccharides; Bcl-2, B-cell lymphoma 2; Bax, Bcl-2-associated $\mathrm{X}$ protein; $\mathrm{C}$, the normal control group; $\mathrm{A}$, the apoptosis model group. 


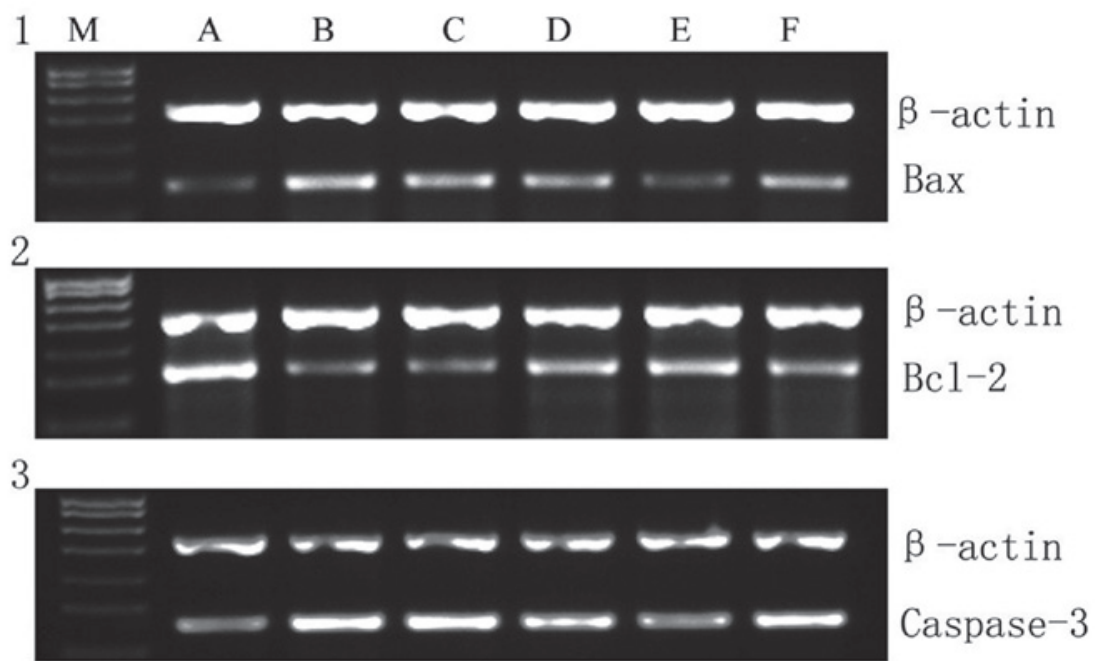

Figure 7. mRNA expression levels of Bax, Bcl-2 and caspase-3 in cultured cerebral cortical neurons were analyzed by polymerase chain reaction and agarose gel electrophoresis. The first column (M) represents the DNA marker. Columns A-F represent: (A) The normal control group; (B) the apoptosis model group; (C) the $0.025 \mathrm{~g} / 1$ RDPS-treated group; (D) the $0.05 \mathrm{~g} / 1$ RDPS-treated group; (E) the $0.10 \mathrm{~g} / 1$ RDPS-treated group; and (F) the $0.25 \mathrm{~g} / 1 \mathrm{RDPS}$-treated group RDPS, Rhizoma Dioscoreae polysaccharides; Bcl-2, B-cell lymphoma 2; Bax, Bcl-2-associated X protein.
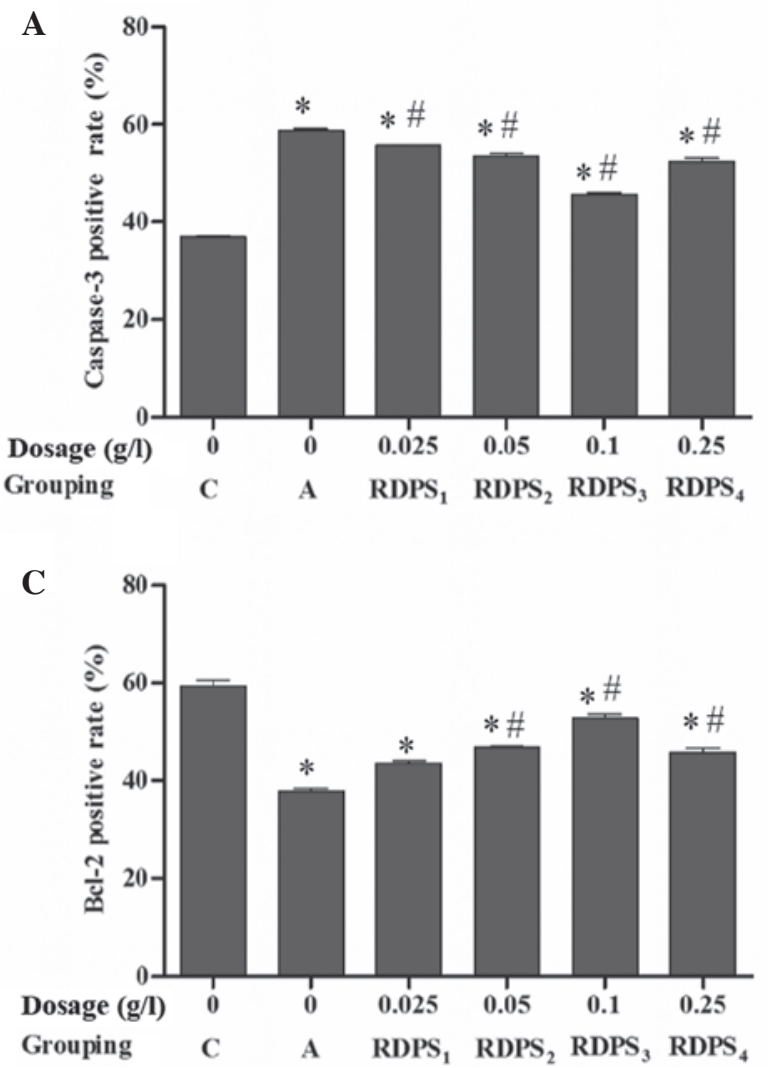
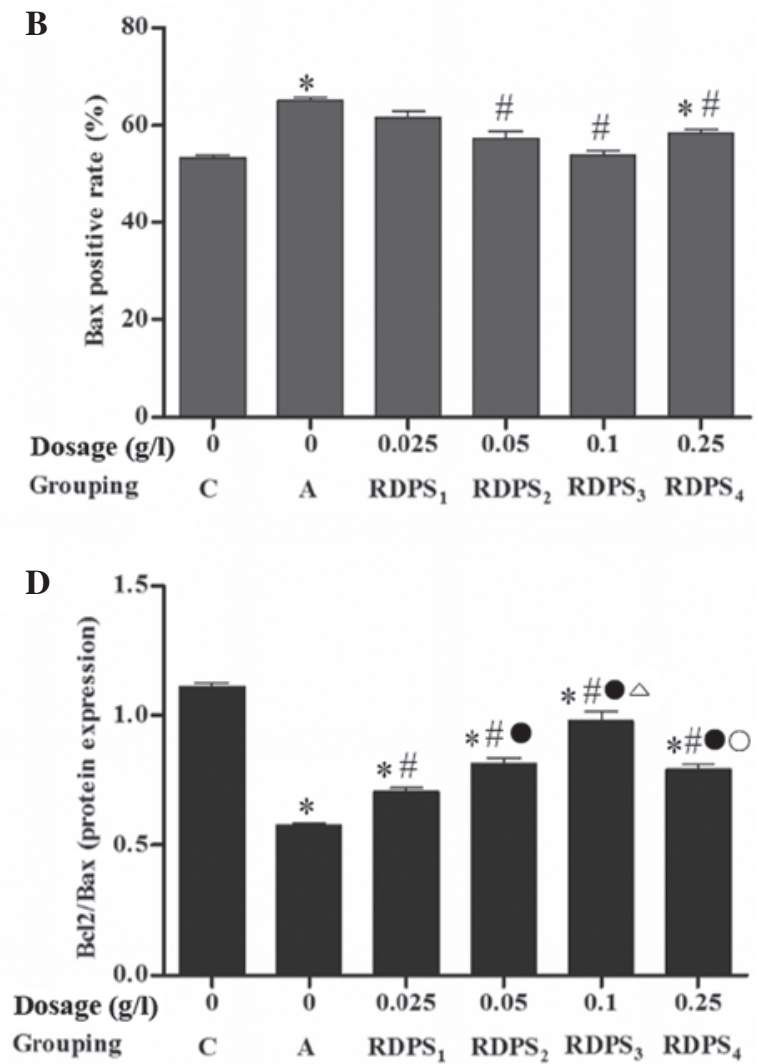

Figure 8. Effects of RDPS on the number of (A) caspase-3-, (B) Bax-, (C) Bcl-2-positive cells and (D) Bcl2/Bax ratio in hypoxic neurons. Neuronal cells cultured under hypoxic conditions for $12 \mathrm{~h}$, under reoxygenating conditions for $24 \mathrm{~h}$, were treated with $0,0.025,0.05,0.10 \mathrm{or} 0.25 \mathrm{~g} / \mathrm{l}$ RDPS. The cells were analyzed using immunocytochemical staining. Data are presented as the mean \pm standard deviation of triplicate experiments. " $\mathrm{P}<0.05$ vs. the normal control group; ${ }^{\prime} \mathrm{P}<0.05$ vs. the apoptosis model group; ${ }^{\circ} \mathrm{P}<0.05$ vs. the RDPS1 group; ${ }^{\circ} \mathrm{P}<0.05$ vs. the RDPS 3 group; ${ }^{\Delta} \mathrm{P}<0.05$ vs. the RDPS2 group. RDPS, Rhizoma Dioscoreae polysaccharides; Bcl-2, B-cell lymphoma 2; Bax, Bcl-2-associated X protein; C, the normal control group; A, the apoptosis model group.

and Bax-positive cells in the hypoxic neurons treated with 0.05-0.25 g/l RDPS (in particular $0.10 \mathrm{~g} / \mathrm{l}$ ) were significantly decreased, as compared with the apoptosis model group $(\mathrm{P}<0.05$; Fig. $8 \mathrm{~A}$ and $\mathrm{B})$. Conversely, the number of Bcl-2-positive cells in the hypoxic neurons treated with
0.05-0.25 g/1 RDPS, particularly with $0.10 \mathrm{~g} / 1$, were significantly increased, as compared with the apoptosis model group $(\mathrm{P}<0.05$; Fig. $8 \mathrm{C}$ ). In addition, following treatment with 0.025-0.25 g/1 RDPS, the ratio of Bcl-2-positive cells:Bax-positive cells was significantly increased in the neurons cultured 
under hypoxia/reoxygenation conditions, as compared with the apoptosis model group $(\mathrm{P}<0.05$; Fig. 8D). These results suggest that decreased hypoxia-induced neuronal cell apoptosis in the RDPS-treated cells may be due to a decrease in the number of caspase-3- and Bax-positive neurons, and an increase in the number of Bcl-2-positive neurons, and the Bcl-2-positive:Bax-positive neuronal ratio.

\section{Discussion}

The pathological occurrence and development of a stroke has previously been associated with the production of ROS, which are produced at the mitochondrial membrane surface. Rhodamine 123 staining is widely used to measure alterations to mitochondrial membrane potential, as it accumulates in the membrane in a manner that is dependent on membrane polarization (12). In the present study, a reduction in Rhodamine $123 \mathrm{MFI}$ and cell viability, and an increase in the rate of cell apoptosis, was detected in the neurons cultured under hypoxic conditions. Treatment with RDPS significantly improved the neuronal cell viability, attenuated mitochondrial injury, enhanced the MFI of the mitochondria and decreased the rate of apoptosis in the hypoxic neurons.

The result of the present study were consistent with the hypothesis that RDPS exerts ROS scavenging activity: RDPS has previously been shown to scavenge ROS and enhance the activities of SOD, GSH-Px and $\mathrm{Na}^{+} / \mathrm{K}^{+}$-ATPase $(4,5,7,8)$. In addition, increased levels of oxidative stress, decreased levels of GSH, catalase, GSH-Px and SOD, and induction of apoptosis, have previously been detected in the brain tissue of a mouse model of global cerebral ischemia/reperfusion (13). Intramitochondrial $\mathrm{Ca}^{2+}$-dependent mitochondrial ROS production is a molecular signal that culminates in the onset of mitochondrial permeability transition (MPT), which may lead to apoptosis $(14,15)$. The opening of the MPT pore may operate as a physiological $\mathrm{Ca}^{2+}$ release mechanism, and may also contribute toward mitochondrial deenergization and the release of pro-apoptotic proteins $(15,16)$. Appukuttan et al $(17)$ detected relocalization of soluble adenylyl cyclase to the mitochondria, which was associated with the initiation of mitochondrial depolarization, cytochrome $c$ release, and caspase-9/-3 cleavage and apoptosis, in cells cultured under hypoxic/reoxygenating conditions.

Whether cell apoptosis occurs is dependent on the balance between the expression of pro- and anti-apoptotic genes, particularly the ratio of Bcl-2:Bax expression levels. In the present study, the expression levels of caspase-3 and Bax were significantly increased, and the expression levels of Bcl-2 and the ratio of $\mathrm{Bcl}-2$ :Bax were significantly decreased, in neurons cultured under hypoxic/reoxygenating conditions. However, RDPS was demonstrated to significantly decrease the expression levels of caspase- 3 and Bax, and increase the expression levels of Bcl-2 and the ratio of Bcl-2:Bax. These results suggested that RDPS may attenuate hypoxia-induced neuronal cell apoptosis by decreasing the mRNA and protein expression levels of apoptosis-initiating genes, and increasing those of anti-apoptotic genes.

Members of the Bcl-2 family have previously been demonstrated to function via conformation-induced insertion into the outer mitochondrial membrane, in order to form channels or pores that regulate the release of apoptogenic factors into the cytosol. Furthermore, Bax heterodimerization with Bcl-2 was shown to neutralize its pro-apoptotic activity. Bax monomers interact to form an oligomeric channel that is permeable to cytochrome $c$. The formation of this channel is blocked by Bcl-2 at multiple sites; however, when Bax is present in excess, the anti-apoptotic activity of $\mathrm{Bcl}-2$ is antagonized, and apoptosis is promoted (18).

Caspase-3 cleaves a protein with deoxyribonuclease activity, and this cleavage activates a cascade of events that culminate in the internucleosomal fragmentation of genomic DNA (16). Previous studies have detected increased expression levels of caspase-3 mRNA and Bax protein, decreased Bcl-2 protein expression levels, and increased cerebral infarct volumes in the brain tissue of rat models of middle cerebral artery occlusion (19-21). Similarly, Huang et al (22) detected significantly increased expression levels of caspase-3/9, and an elevated neurocyte apoptosis rate, in the brain tissue of a C57BL/6 mouse model of bilateral common carotid artery occlusion.

In conclusion, the present study demonstrated that RDPS was able to improve the viability of hypoxic neuronal cells, which may be associated with its effects on mitochondrial function and the expression levels of apoptosis-regulating proteins. In particular, RDPS was able to decrease the mRNA and protein expression levels of Bax and caspase-3, and increase the mRNA and protein expression levels of Bcl-2, culminating in an increase in the ratio of Bcl-2:Bax in hypoxic neurons. The results of the present study suggest that RDPS may be considered for the prevention and treatment of ischemic cerebral diseases and ageing.

\section{Acknowledgements}

The present study was supported by the Social Development Key Research Project of the Jiangxi Provincial Department of Science and Technology (grant no. 2007BS22602).

\section{References}

1. Yuan SL: Research advances on chemical compositions and bioactivities of Dioscorea opposita Thunb. Shi Pin Yan Jiu Yu Kai Fa 29: 176-179, 2008 (In Chinese).

2. Ko MK and Hong KE: Evaluation of the safety of Sanyak (Dioscoreae rhizoma) pharmacopuncture according to the extraction method: A double-blind randomized controlled trial. J Acupunct Meridian Stud 6: 41-51, 2013.

3. Sun S, Zhao J, Guan ST, Zhang H and Liu X: Effect of yam polysaccharide on the contents of free radicals and tumor necrosis factor $\alpha$ in mice of CCl4-induced liver injury. Shan Xi Yi Ke Da Xue Xue Bao 42: 452-454, 2011 (In Chinese).

4. Zan T, Tao J and Wang SR: The antiaging effects of water-soluable polysaccharide from Rhizoma Dioscorea opposita on mice. Yao Xue Jin Zhan 23: 356-360, 1999 (In Chinese).

5. Tang W,Zhu ML and Song MH: Experimental studies on the effect of Chinese yam polysaccharides on anti-aging of mice. Huang Gang Zhi Ye Ji Shu Xue Yuan Yuan Bao 4: 23-25, 2002 (In Chinese).

6. Lee JM, Namgung U and Hong KE: Growth-promoting activity of Sanyak (Dioscoreae rhizoma) extract on injured sciatic nerve in rats. J Acupunct Meridian Stud 2: 228-235, 2009.

7. Shang XY, Ren J, Cao G, Xu CL, Niu WN and Qin CG: Preparation and antioxidant properties of polysaccharides from Dioscorea opposita thumb roots. Hua Xue Yan Jiu 21: 72-76, 2010 (In Chinese).

8. Xu XQ, Liu ZF, Huo NR, Zhao Y, Tian X and Lei TT: In vitro anti-oxidation capacity and immunomodulatory efficacy in mice of yam polysaccharide. Zhong Guo Liang You Xue Bao 27: 42-46, 2012 (In Chinese). 
9. Kesaraju S, Nayak G, Prentice HM and Milton SL: Upregulation of Hsp72 mediates anoxia/reoxygenation neuroprotection in the freshwater turtle via modulation of ROS. Brain Res 1582: 247-256, 2014

10. Lee KY, Bae ON, Weinstock S, Kassab M and Majid A: Neuroprotective effect of asiatic acid in rat model of focal embolic stroke. Biol Pharm Bull 37: 1397-1401, 2014.

11. Hu WX, Xiang Q, Wen Z, He D, Wu XM and Hu GZ: Neuroprotective effect of Atractylodes macrocephalaon polysaccharides in vitro on neuronal apoptosis induced by hypoxia. Mol Med Rep 9: 2573-2581, 2014.

12. Huang M, Camara AK, Stowe DF, Qi F and Beard DA: Mitochondrial inner membrane electrophysiology assessed by rhodamine-123 transport and fluorescence. Ann Biomed Eng 35: 1276-1285, 2007

13. Ciftci O, Oztanir MN and Cetin A: Neuroprotective effects of $\beta$-myrcene following global cerebral ischemia/reperfusion-mediated oxidative and neuronal damage in a C57BL/J6 mouse. Neurochem Res 39: 1717-1723, 2014.

14. Kim JS, Wang JH and Lemasters JJ: Mitochondrial permeability transition in rat hepatocytes after anoxia/reoxygenation: Role of $\mathrm{Ca}^{2+}$-dependent mitochondrial formation of reactive oxygen species. Am J Physiol Gastrointest Liver Physiol 302: G723-G731, 2012.

15. Christophe $\mathrm{M}$ and Nicolas S: Mitochondria: A target for neuroprotective interventions in cerebral ischemia-reperfusion. Curr Pharm Des 12: 739-757, 2006.
16. Martin LJ: Mitochondrial and cell death mechanisms in neurodegenerative diseases. Pharmaceuticals (Basel) 3: 839-915, 2010.

17. Appukuttan A, Kasseckert SA, Micoogullari M, Flacke JP Kumar S, Woste A, Abdallah Y, Pott L, Reusch HP and Ladilov Y: Type 10 adenylyl cyclase mediates mitochondrial Bax translocation and apoptosis of adult rat cardiomyocytes under simulated ischaemia/reperfusion. Cardiovasc Res 93: 340-349, 2012.

18. Gustafsson AB and Gottlieb RA: Bcl-2 family members and apoptosis, taken to heart. Am J Physiol Cell Physiol 292: C45-C51, 2007.

19. Yang J, Li J, Lu J, Zhang Y, Zhu Z and Wan H: Synergistic protective effect of astragaloside IV-tetramethylpyrazine against cerebral ischemic-reperfusion injury induced by transient focal ischemia. J Ethnopharmacol 140: 64-72, 2012.

20. He B, Chen P, Yang J, Yun Y, Zhang X, Yang R and Shen Z: Neuroprotective effect of $20(\mathrm{R})$-ginsenoside $\operatorname{Rg}(3)$ against transient focal cerebral ischemia in rats. Neurosci Lett 526: 106-111, 2012.

21. Zhang G, Liu A, Zhou Y, San X, Jin T and Jin Y: Panax ginseng ginsenoside- $\mathrm{Rg} 2$ protects memory impairment via anti-apoptosis in a rat model with vascular dementia. J Ethnopharmacol 115: 441-448, 2008

22. Huang XP, Tan H, Chen BY and Deng CQ: Astragalus extract alleviates nerve injury after cerebral ischemia by improving energy metabolism and inhibiting apoptosis. Biol Pharm Bull 35: 449-454, 2012. 